\section{Aneurysms of the superficial venous system: classification and treatment}

\author{
Ronald G. Bush, Peggy Bush \\ Vein Experts, Palm City, FL, USA
}

\section{Abstract}

Superficial venous aneurysms are rarely described and they may remain indolent or become the source for pulmonary emboli. A system of classification and treatment protocol according to size and location is proposed.

Three hundred thirty patients were evaluated for symptomatic venous disease (C2-C6) over a 2-year period. A proposed designation for venous aneurysm is described. Patients fulfilling this criterion are described in reference to site of involvement, histologic findings, and method of treatment. Five percent of patients met the criteria for venous aneurysm. Nine aneurysms of the greater saphenous vein were identified. Three aneurysms were proximal to the subterminal valve and the rest were distal. Six aneurysms of the anterior accessory greater saphenous vein (AAGSV) were identified. Three aneurysms of the AAGSV spontaneously thrombosed. Two patients presented with aneurysms of the small saphenous vein. Histology revealed thickened intima, smooth muscle and adventitia. Aneurysm designation relates to diameter of normal and contiguous vein. All superficial venous aneurysms in close proximity to the junction of the femoral or popliteal vein should be ligated. Classification of venous aneurysms should include the AAGSV, which may present with spontaneous thrombosis.

\section{Introduction}

Superficial venous aneurysms secondary to venous hypertension are more common than reported in the literature. The subject of this report are those aneurysmal dilations occurring within the fascial compartments of the greater saphenous vein (GSV), small saphenous vein (SSV) or the anterior accessory saphenous vein (AAGSV). Many reports describing venous aneurysms include multiple sites in both the deep and superficial system. Pascarella et al. ${ }^{1}$ described their experience with venous aneurysms of the lower extremity and proposed a classification based on location in the superficial system.

A consensus as to the exact definition of a venous aneurysm secondary to chronic venous disease does not exist. Even histological findings in venous aneurysms vary in reported studies. Histological discrepancies may represent the comparison of primary venous aneurysms to aneurysms secondary to venous hypertension. ${ }^{2,3}$ The duration of hypertension may also become a factor in the histological findings.

Most reported cases with embolic events associated with venous aneurysms are located in the deep system. ${ }^{4}$ However, there are also reports in the literature of emboli originating in the superficial venous system. Embolic sources have included the GSV, AAGSV, SSV and other superficial veins of the neck and upper extremity. ${ }^{5-9}$ The purpose of this study is to examine our experience with superficial venous aneurysms secondary to venous hypertension in regard to presenting symptoms, treatment, potential complications, and histological findings.

\section{Materials and Methods}

Three hundred thirty patients with symptomatic venous disease (C2-C6) were evaluated over a 2-year period. Of these patients, eighteen met the proposed criteria for venous aneurysm; the vein size was $3 x$ the normal respective vein diameter and $2 x$ the size of the contiguous vein. Five patients were male, 13 female with a median age of 55 . The basal metabolic rate was greater than 30 in only two patients.

\section{Results}

A total of 9 aneurysms were located in the GSV. Three aneurysms were located at the sapheno-femoral junction (SFJ). Aneurysms of the SFJ are localized between the terminal and subterminal valve and include the junctional branches (Figure 1). Two aneurysms were located just distal to the sub-terminal valve and 4 were located in the distal GSV. Six patients presented with aneurysms of the AAGSV. Three of these patients presented with acute thrombosis of the aneurysm and associated superficial branches (Figure 2). Two aneurysms of the SSV were identified. One involved the sapheno-popliteal junction (SPJ) and one aneurysm was located at the mid SSV. One patient presented with an aneurysm of the posterior medial thigh circumflex branch.

\section{Discussion}

No consensus exists on what the criteria for the designation of venous aneurysms should be. Pascarella ${ }^{1}$ used the criteria of $1.5 \mathrm{x}$ the size
Correspondence: Peggy Bush,

Vein Experts, 1257 SW Martin Hwy \#768, Palm City, FL 34991, USA. E-mail: pbush@veinexperts.org

Key words: venous aneurysm, superficial venous disease, chronic venous disease, venous hypertension.

Received for publication: 6 July 2014

Revision received: 18 October 2014.

Accepted for publication: 20 October 2014.

This work is licensed under a Creative Commons Attribution 3.0 License (by-nc 3.0).

(C) Copyright R.G. Bush and P. Bush, 2014

Licensee PAGEPress, Italy

Veins and Lymphatics 2014; 3:4503

doi:10.4081/vl.2014.4503

of the contiguous vein and this is also the measurement used to describe an arterial aneurysm. Many valvular dilatations in the venous system could meet this definition. Other criteria for venous aneurysmal designation have included $3 \mathrm{x}$ the size of the normal vein, or $2 \mathrm{x}$ the size of the contiguous vein. ${ }^{10} \mathrm{~A}$ more rigorous criteria would include both vein diameter compared to normal and contiguous vein. Based on histologic studies by the authors of both extrafascial varicose vein segments and those segments meeting the proposed size requirement for a venous aneurysm, the histologic findings are very similar (Figure 3). In both the varicose segment and the aneurysmal segment, there is smooth muscle hypertrophy to varying degrees. The intima is thickened as well as the adventitia with collagen infiltration. These findings are consistent with the end results of persistent venous hypertension; muscle hypertrophy and collagen breakdown with abnormal synthesis of proteins. As the hypertension persists, more dilatation may occur with elongation of muscle fibers and disruption of the internal and external elastic membrane. Histological findings may vary somewhat depending on the crosssectional study in the region of the valve, or if saccular dilation occurs as opposed to circumferential involvement. Similar changes have been described previously in histological studies of the refluxing GSV at the SFJ. ${ }^{11}$ These changes only occur at the location of nonfunctioning venous valves. Venous aneurysms of vessels not associated with chronic venous hypertension (primary or congenital) demonstrate thinning of the medial layer of the venous wall. ${ }^{12}$

The etiology of venous aneurysmal dilatation secondary to chronic venous disease is the same as for varicosed segments; persistent venous hypertension and abnormal valve function. One theory portends a turbulent flow pat- 
tern associated with a dysfunctional valve. ${ }^{13}$ Over a prolonged period of time, the forces associated with this phenomenon can lead to the observed abnormal tissue response. Turbulent flow is often visualized near the valve sinus. Aneurysmal designation becomes important because increasing vein diameter makes stasis more likely, and potential thromboembolism more probable depending on the specific location of the aneurysm. Also conventional treatment may have to be altered due to aneurysmal size or location as described in the section describing treatment. Only aneurysmal dilatation in the respective fascial sheath alters treatment or outcome potential.

Aneurysms of the GSV have been classified as to location distal to the subterminal valve and in the distal thigh GSV. ${ }^{1}$ However, aneurysmal dilatation may also include the junctional branches and the SFJ itself proximal to the subterminal valve. The aneurysmal dilation proximal or distal to the subterminal valve should be classified separately since treatment is different. The authors propose that aneurysmal dilations of the SFJ (includes junctional branches) be classified as Ia. Aneurysmal dilatation distal to the subterminal valve should be classified as Ib. For aneurysmal dilations of the distal GSV occurring in the region of the lower thigh perforators or upper calf paritibial perforator, a classification of II correlates with Pascarella's ${ }^{1}$ classification. Four patients in our series had aneurysmal dilatation of the distal GSV without proximal involvement. Three patients presented with aneurysms in the region of Hunter's canal. One aneurysm was located in the proximity of a calf paratibial perforator. In both regions described, a valve is commonly present. When aneurysmal dilatations exist in both the proximal and distal GSV then they are classified as either IIIa or IIIb depending on location of aneurysmal dilation at the SFJ.

Aneurysmal dilations have been reported in the SSV. ${ }^{1,14}$ Pascarella labeled this as type IV. ${ }^{1}$ In our series, aneurysms were noted at the SPJ and the mid SSV. The authors propose IVa to refer to the location at the SPJ and IVb to refer to a more distal location.

Aneurysmal dilation of the AAGSV has rarely been described. In reviewing the literature, only one isolated case report of pulmonary emboli in a young male originating from the AAGSV was found. In our series, 6 patients were identified with aneurysmal dilatation of the AAGSV, accounting for $33 \%$ of the patients with aneurysmal findings. Three of these patients presented with acute superficial thrombophlebitis and thrombosis of the origin of the aneurysmal AAGSV.

One of the 6 patients had an aneurysmal dilation of the AAGSV at mid-thigh with a normal diameter proximal AAGSV. The authors proposed that aneurysmal dilation of the prox- imal AAGSV be labeled as Va and distal location be labeled as $\mathrm{Vb}$.

One patient had an aneurysmal dilation of the posterior medial thigh circumflex branch.

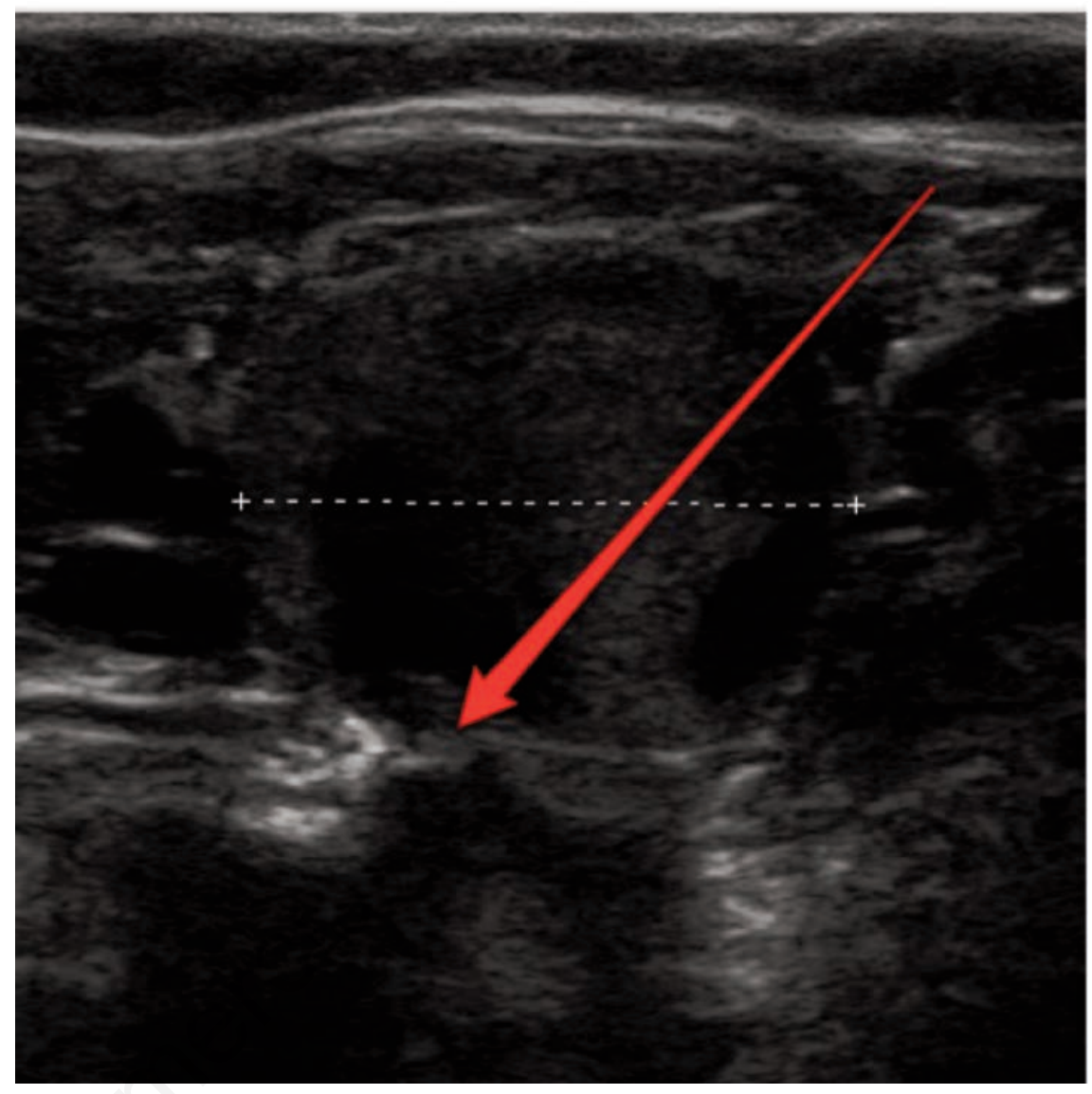

Figure 1. Aneurysm is between the terminal and pre-terminal valve and includes the junctional branches - Type I.

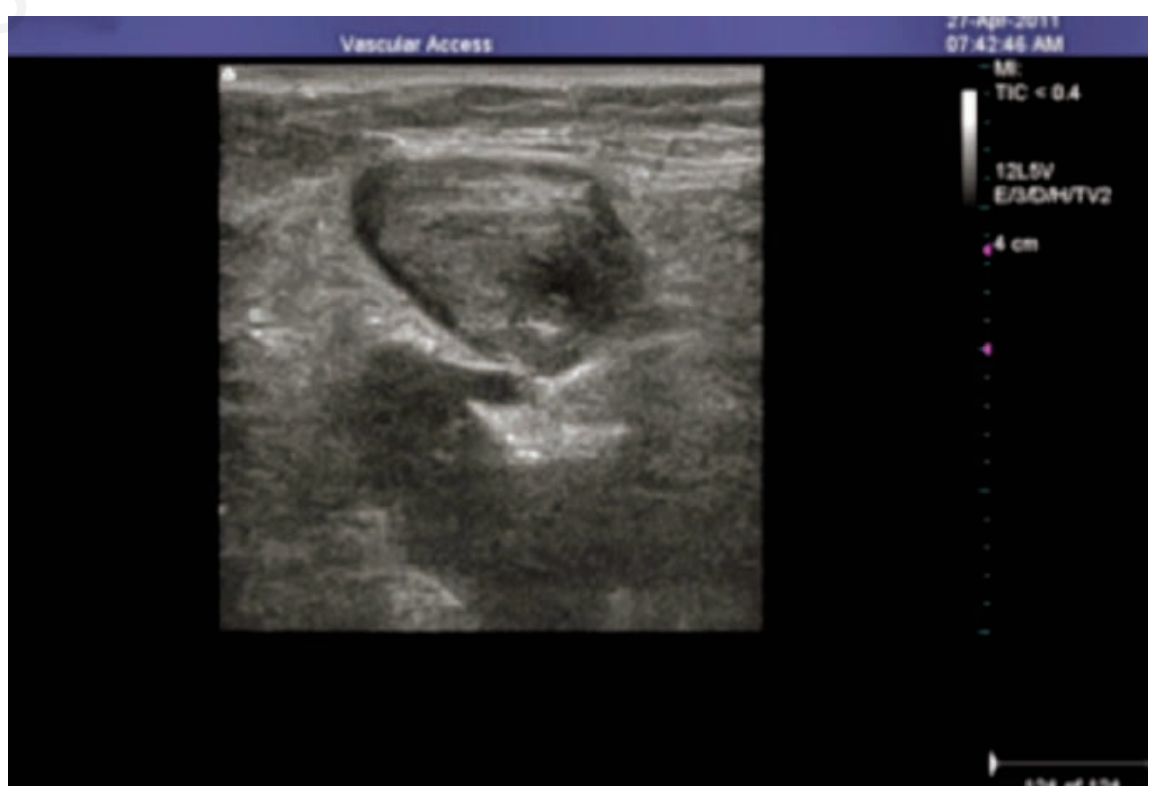

Figure 2. Thrombus in anterior accessory greater saphenous vein aneurysm. Note small communication to sapheno-femoral junction.
The authors propose that aneurysmal dilation not fulfilling criteria $\mathrm{I}-\mathrm{V}$, be classified into a general group labeled VI.

Aneurysmal dilatations of the superficial

.


system (Ia, IVa, Va) have been reported as foci of pulmonary emboli. The true incidence is unknown, but the occurrence is much lower than from the venous aneurysms of the deep system. Most foci of embolization from the deep system originate from the popliteal vein. ${ }^{4}$ Histologically, popliteal and femoral aneurysms are distinct from those occurring after valvular dysfunction in the superficial system, as would be expected since the etiology of these aneurysms is different.

\section{Treatment}

Superficial venous aneurysms are managed in accordance with specific location. Aneurysmal dilatations that involve the SFJ (la) pose a significant threat as a source of embolization. Patients presenting with spontaneous thrombosis at the SFJ should be immediately anticoagulated. ${ }^{15}$

For those aneurysmal dilatations found on ultrasound that involve the true SFJ, high ligation of the GSV should be considered. Standard thermal ablative techniques may increase the risk of clot propagation. A large diameter vein with minimal outflow has a higher propensity for thrombus formation.

Aneurysms of the GSV distal to the subterminal valve (Ib) may be treated with conventional thermal ablative techniques or foam sclerotherapy. Since normal inflow persists, the risk of clot propagation is low. Aneurysms of the distal GSV (II) may be treated with surgical or endovascular ablative techniques. The method of treatment depends on the distance from the skin and size of the aneurysm. Aneurysms that are very superficial and not resected will clot after treatment. A resultant thrombus can take months to regress and be cosmetically unacceptable and a source of prolonged discomfort.

Aneurysms involving the SSV are also treated according to the specific location. All aneurysms involving the SPJ (IVa) should be resected. IVa aneurysms are anatomically similar to saccular aneurysms of the popliteal vein. Thermal ablation distal to these IVa aneurysms may induce clotting of the SPJ with clot propagation or embolization. Aneurysmal dilatations classified as IVb are treated in a manner similar to that described for distal GSV aneurysms.

Aneurysms of the AAGSV are treated according to location and presenting symptoms. For those that present with spontaneous thrombosis, anticoagulation should be considered especially if the communication between the AAGSV and GSV is large ( $>4 \mathrm{~mm}$ ). For nonthrombosed AAGSV aneurysms, 3 treatment options exist. Surgical resection or ligation may be warranted in those situations where a large communication exists between the AAGSV and the GSV or both are dilated. When the communication is small, either distal abla- tion of the AAGSV trunk or foam sclerotherapy are options. Two in this group were treated with foam sclerotherapy of the AAGSV aneurysmal dilatation and distal phlebectomy. One patient was treated with thermal ablation of the AAGSV. The aneurysmal dilatation thrombosed with no adverse sequalae. Out of the 3 aforementioned patients the communication with the GSV was $<4 \mathrm{~mm}$.

\section{Conclusions}

Aneurysmal dilations of the AAGSV, GSV, and SSV are similar histologically to varicosed segments of branched veins. The etiology is also similar to turbulent flow at a valve that is dys- functional which may occur for a variety of reasons; volume overload secondary to pregnancy, hereditary factors and venous hypertension transmitted from the deep system. Any location in the superficial system that contains a valve under less than optimal conditions may become aneurysmal.

Aneurysmal dilatations in the superficial system should be classified with regard to both normal vein diameter and size relationship to contiguous vein. Classification of aneurysmal dilatation should include location corresponding to valves in the GSV. Aneurysms of the SFJ, SPJ and origin of the AAGSV pose potential threat of thromboembolization.

In addition, the AAGSV aneurysmal dilatation should be added to the classification. AAGSV aneurysms are more common than

\section{A}

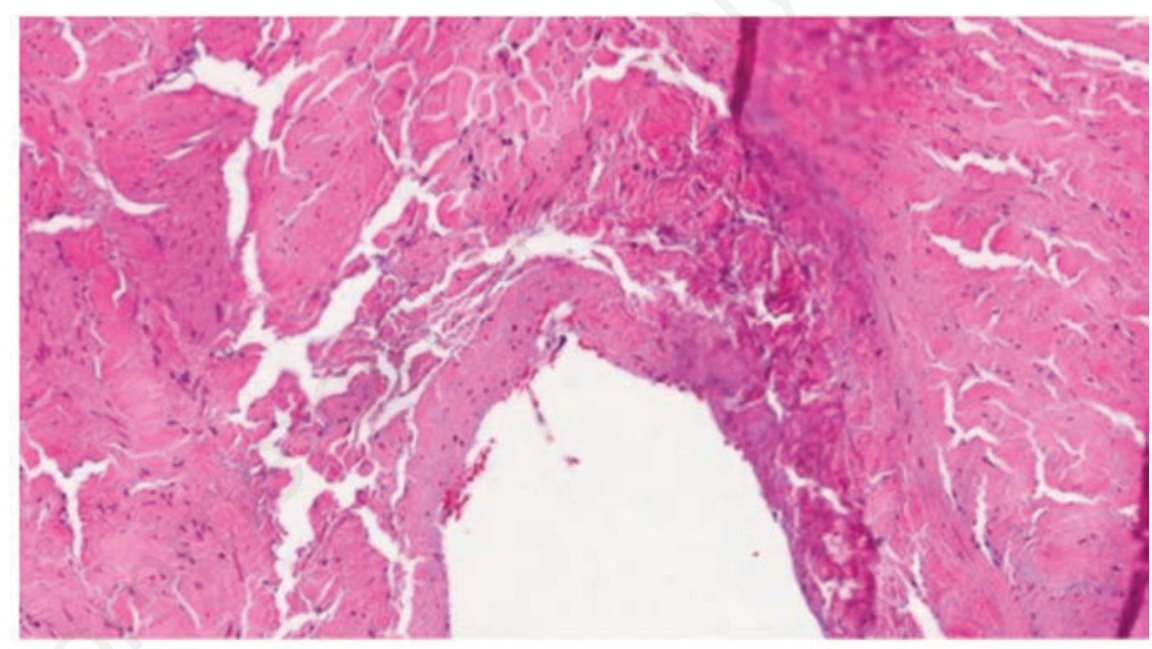

B

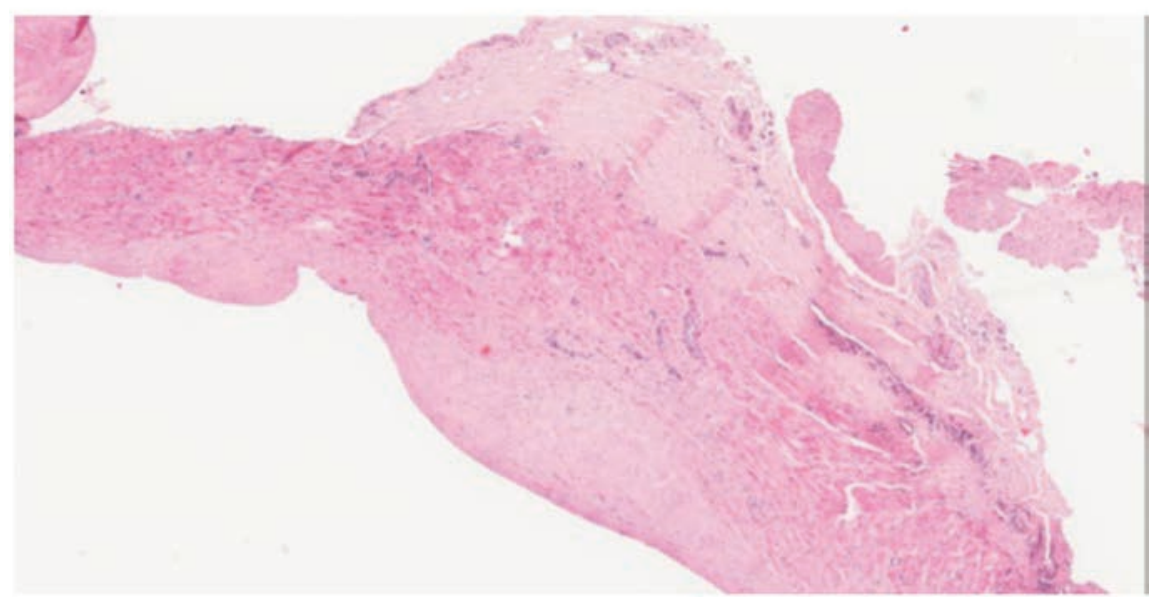

Figure 3. A) Hematoxylin and eosin (H\&E) stain of aneurysm of greater saphenous vein (GSV). Note thickened intima and muscle hypertrophy; B) H\&E stain of branch varicosity. Similar histological findings to aneurysmal dilatation of GSV, anterior accessory greater saphenous vein, and small saphenous vein. 
reported and may present as acute thrombosis of distal varices or as a source of pulmonary emboli.

\section{References}

1. Pascarella L, Al-Tuwaijri M, Bergan JJ, Mekenas LM. Lower extremity superficial venous aneurysms. Ann Vasc Surg 2005;19:69-73.

2. Seo SH, Kim MB, Kwon KS, et al. Primary venous aneurysm of the superficial venous system. Angiology 2008;59:593-8.

3. Wali MD, Dewan M, Eld RA. Histopathological changes in the wall of varicose veins. Int Angiol 2003;22:188093.

4. Sessa C, Nicolini P, Perrin M, et al. Management of symptomatic and asymptomatic popliteal venous aneurysms: A retrospective analysis of 25 patients and review of the literature. J Vasc Surg
2000;32:902-12.

5. Gabrielli R, Rosati M, Millarelli M, et al. Pulmonary emboli due to venous aneurysm of extremeties. Eur J Vasc Med 2011;40:327-32.

6 Gillespie D, Villavicencio J, Gallagher C, et al. Presentation and management of venous aneurysms. J Vasc Surg 1997; 26:845-52.

7. Marcucci G, Accrocca F, Antignani P, Siani A. An isolated aneurysm of the thigh anterolateral branch of the greater saphenous vein in a young patient presenting as an inguinal hernia. Interact CardioVasc Thorac Surg 2010;10:654-5.

8. Wallace J, Baril D, Chaer R. Upper extremity venous aneurysms as the source of pulmonary emboli. Ann Vasc Surg 2013;27: 240.

9. Cavezzi A, Labropoulos N, Partsch, et al. Duplex ultrasound investigation of the veins in chronic venous disease of the lower limbs-UIP consensus document. Part II. Anatomy. Vasa 2006;35:62-71.
10. Gabrielli R, Rosati MS, Siani A, Irace L. Management of symptomatic venous aneurysm. Sci World J 2012;6:386478.

11. Stucker M, Krey T, Rochling A, et al. The histomorphologic changes at the saphenofemoral junction in varicosis of the greater saphenous vein. Vasa 2009;29:41-6.

12. Irwin C, Synn A, Kraiss L, et al. Metalloproteinase expression in venous aneurysms. J Vasc Surg 2008;48:1278-84.

13. Tibbs DJ. Varicose veins and related disorders. London: Butterworth Heinemann; 1992. pp 15-16.

14. Chen S, Clouse W, Bowser A, Rasmussen T. Superficial venous aneurysm of the small saphenous vein. J Vasc Surg 2009;50:644-7.

15. Kearon C, Akl E, Comerota A, et al. Antithrombotic therapy for VET disease. Antithrombotic therapy and prevention of thrombosis, $9^{\text {th }}$ ed. American college of chest physicians evidence-based clinical practice guidelines. Chest 2012;141: e419S-94S. 